\title{
Long non-coding RNA MT1JP exerts anti-cancer effects in breast cancer cells by regulating miR-92-3p
}

\author{
Haiming $\mathrm{Wu}^{1}$ and $\mathrm{Sa} \mathrm{Li}^{2}$ \\ ${ }^{1}$ Department of Breast Surgery, Shanxi provincial Cancer Hospital, Shanxi Medical University, Taiyuan, 030013, Shanxi, China \\ ${ }^{2}$ Department of ICU, 985 Hospital of PLA Joint Logistics Support Force, Taiyuan, 030001, Shanxi, China
}

\begin{abstract}
MT1JP is a LncRNA that is reportedly involved in gastric cancer development, but a biological role and mechanism for MT1JP in breast cancer is unknown. Quantitative RT-PCR was performed to detect the level of MT1JP and miR-92a-3p, and Western blotting assays ware performed to measure the expression of CDK2, cyclinE1, P21, CD151, CD147, MMP2 and MMP9 in breast cells. Subsequently, cell viability was analyzed with CCK-8 assay. Cell migration and invasion were analyzed with Transwell and Scratch Test, respectively. The results demonstrated that MT1JP was significantly down-regulated in breast cells. Additionally, we found that overexpression of MT1JP in breast cancer cells significantly inhibited cell proliferation, migration and invasion, and regulate the expression of CDK2, cyclinE1 and P21. We then investigated a possible mechanism for these results, MT1JP significantly inhibited CD151, CD147, MMP2 and MMP9 protein expression in breast cancer cells. Moreover, we found that MT1JP binds to and negatively regulates miR-92-3p, which is known to be an oncogene in some human cancers. Our data indicate that MT1JP functions as an anti-tumor LncRNA and downregulates miR-92-3p, CD151 and CD147, and may serve as a novel diagnostic and therapeutic marker in breast cancer.
\end{abstract}

Key words: MT1JP — Breast cancer — Proliferation — Migration — Invasion

\section{Introduction}

The incidence of breast cancer increasing year by year, which is one of the most common malignant tumors worldwide occurred mainly in female patients (Siegel et al. 2017; Lu et al. 2018) . Basic research and clinical treatment of breast cancer have made great progress, nevertheless, the prognosis remains poor (Liu et al. 2017; Jastrzebski et al. 2018). Although the chemotherapy is the most common way for cancer treatment, the drug assistance is also a big issue. New kind of drugs like HAp Doped with $\mathrm{Ce} 2 \mathrm{O} 3$ also has effects on breast cancer (Thirumamagal et al. 2018). In addition, poor prognosis can be ascribed to incomplete diagnosis, high recurrence rate, and therapy resistance, and the adverse effects after combined treatment (Burki 2018; Liu et al. 2018; Ulker et al. 2018). Therefore, elucidating the potential mo-

Correspondence to: Sa Li, Department of ICU, 985 Hospital of PLA Joint Logistics Support Force, No. 30, Middle section of Qiaodong Street, Yinze District, Taiyuan, 030013, Shanxi, China E-mail: lisa_264@163.com lecular mechanisms, and developing novel biotherapeutics target for this clinically intractable tumor is very important.

LncRNAs are known as a type of functional RNA which are longer than $200 \mathrm{nts}$, lacking in open reading frames and the ability to code for proteins (Thomson and Dinger 2016). Many recent studies have reported that LncRNAs were closely related to human diseases, including cancers such as lung, breast, and gastric cancer (Nagini 2017). LncRNA MT1JP is a gene consisting of a few homologous protein-coding genes in the metallothionein family located on chromosome 16. MT1JP is originally discovered to regulate the tumor suppressor p53 protein expression and p53-related signaling pathway (Liu et al. 2016). MT1JP expression is aberrantly decreased both in gastric cancer tissues and cell lines, which is also closely related to lymphatic metastasis, progression of TNM stage, and shorter survival times (Zhu et al. 2018). Besides, in vitro analysis has revealed that MT1JP promotes cell apoptosis, and alleviates cell proliferation, migration and invasion in gastric cancer. Moreover, MT1JP could also suppress tumor growth and metastasis in vivo (Lv et al. 2018; Xu et al. 2018; Zhang et al. 2018). Whether MT1JP is abnormally 
functioned in biological alteration and further mechanisms in breast cancer remains unknown.

CD147 is a transmembrane glycoprotein, which belongs to a member of the human immunoglobulin superfamily and overexpressed in many tumors including breast cancer (Li et al. 2009). As an inducer of matrix metalloproteinase (MMPs), CD147 plays a critical role in cancer proliferation, invasion and metastasis by remodeling the extracellular matrix through activation of MMPs (Pinheiro et al. 2015). The tetraspanin CD151 is an important member of the tetraspanin family. CD151 contributes to integrin-dependent cell adhesion and motility by directly interacting with laminin-binding integrins ( $\alpha 3 \beta 1, \alpha 6 \beta 1, \alpha 6 \beta 4$, and $\alpha 7 \beta 1$ ) (Zhang et al. 2002). Upregulation and overexpression of CD151 are found in many tumor types. Previous studies have confirmed that the expression of CD151 increased markedly in breast cancer tissues compared with that in normal breast tissues. CD147 and MMP26 might be regulated by CD151 in a cell type specific manner (Liu et al. 2015). Further studies revealed that LncRNA could act as a "Sponge" to regulate microRNA (miRNA) in breast or other cancer cells, which targets CD151 or CD147 and regulates downstream functions (Han et al. 2013; Li et al. 2017). Therefore, the relationship among MT1JP, CD151 and/or CD147 in breast cancer were urgently investigated.

In this study, we found that MT1JP mediated breast cell line proliferation, invasion and migration by negatively regulating miR-92a-3p. To the best of our knowledge, these data are the first indication that MT1JP plays an important tumor-suppression role in breast cancer.

\section{Methods}

\section{Cell culture}

The breast epithelial cell line MCF-10A, human breast cancer cell lines MCF-7 and MDA-MB-231 were purchased from American Type Culture Collection (ATCC, USA), and routinely cultured in Dulbecco's modified Eagle's medium (DMEM) (Invitrogen) with L-glutamine and 10\% (v/v) fetal bovine serum (FBS; ExCell Bio). All cells were maintained at $37^{\circ} \mathrm{C}$ in a $5 \% \mathrm{CO}_{2}$ humidified atmosphere.

\section{Cell transfection}

The MT1JP overexpression plasmid was purchased from $\mathrm{Ge}$ nePharm (Shanghai, China). The MT1JP was also purchased from Shanghai Gene Pharma Co. Ltd (Shanghai, China). The sequence was amplified and inserted into the pcDNA3.1 (+) vector. For transfection, the MCF-7 and MDA-MB-231 cells were grown in 6-well plates to $50-70 \%$ confluence, then were transfected with the indicated molecules using Lipofectamine 3000 (Thermo) according to the manufacturer's instructions. Forty-eight hours after transfection, cells were ready for the following experiments.

Reverse transcription-quantitative polymerase chain reaction $(R T-q P C R)$

Total RNA from tissues and cells was isolated using Trizol reagent (Invitrogen, Carlsbad, USA) according to the manufacturer's instructions. RNA was reverse transcribed with the PrimeScript RT Reagent Kit (Invitrogen, USA), quantitative reverse transcription PCR (qRT-PCR) was performed using SYBR Premix Ex Taq (TaKaRa, China), following the manufacturer's instructions. GAPDH was used as an internal LncRNA and microRNA control. The primer sequences were as follows: MT1JP, forward: 5'-TACCGAGCTCG-GATCCTTGCGGTCTCTCCATTTATCG-3', reverse: 5'-TACCGAGCTCGGATCCTTGCGGTCTCTCCATTTATCG-3'; miR-92a-3p, forward: 5'-GGGGCAGTTATTGCACTTGTC-3', reverse: 5'-CCAGTGCAGGGTCCGAGGTA-3'; GAPDH, forward: 5'-GCACCGTCAAGGCTGAGAAC-3', reverse: 5'TGGTGAAGACGCCAGTGGA-3'. We performed qRT-PCR using the ABI PRISM 7500 PCR System (Applied Biosystems, Foster City, CA, USA) according to the manufacturer's instructions. $2^{-\Delta \Delta \mathrm{Ct}}$ method was used in each sample as relative quantification (Livak and Schmittgen 2001).

\section{Cell proliferation assay}

After transfection with the empty vector or pcDNA-MT1JP, we used the Cell Counting Kit-8 (CCK-8; Dojindo Molecular technologies, Inc., Kyushu, Japan) according to the manufacturer's protocol to measure cell proliferation. Briefly, we plated MCF-7 or MDA-MB-231 cells into 96-well plates $\left(2 \times 10^{3}\right.$ cell/well $)$ respectively. And $10 \mu \mathrm{l} \mathrm{CCK-} 8$ were added into each well at $24,48,72$ and $96 \mathrm{~h}$ by incubating at $37^{\circ} \mathrm{C}$. The optical density (OD) was measured at $450 \mathrm{~nm}$ on an enzyme-linked immunosorbent assay reader.

\section{Cell invasion assay}

Cell migration assays were performed using 24-well culture plates inserted with $8-\mathrm{mm}$ pore size culture (Transwell; Falcon, BD Biosciences). The lower chamber was filled with $600 \mu \mathrm{l}$ DMEM containing 10\% FBS. MCF-7 or MDA-MB-231 cells $\left(1 \times 10^{5}\right.$ cell/well $)$ were seeded to the upper chamber respectively. After $24 \mathrm{~h}$ incubation, the number of the bottom well cells were counted by counting chamber.

\section{Cell migration assay}

For wound healing assay, MCF-7 or MDA-MB-231 cells were seeded in 6 -well plates $\left(5 \times 10^{5}\right.$ cells/well). A pipette tip was used to create a wound after $48 \mathrm{~h}$ transfection. The cells 
were then cultured in serum-free medium. Cell migration was determined by detecting the average distance of growing cells migrated into wound surface under an inverted microscopy at $0 \mathrm{~h}$ and $24 \mathrm{~h}$.

\section{Western blot analysis}

The cells and samples were collected and lysed at $48 \mathrm{~h}$ after treatment using RIPA buffer. Identical amounts of proteins were resolved by 10-15\% SDS-PAGE and then transferred to a PVDF membrane (Millipore Corporation, Billerica, MA, USA). The membrane was incubated with specific antibodies (CDK2, cyclinE1, P21, CD151, CD147, MMP2 and MMP9; Cell Signaling Technology Inc.) and peroxidase-conjugated secondary antibodies (KPL, Gaithersburg, MD, USA) at $4^{\circ} \mathrm{C}$ overnight. Chemiluminescence (Milipore Corporation) and densitometry analysis (ImageJ software) were applied to measure the protein expression.

\section{Statistical analysis}

Data were expressed as the mean \pm standard deviation (SD). SPSS 17.0 statistical software (SPSS, Inc., Chicago, IL, United States) was used for all statistical analyses. Comparisons were analyzed by one-way ANOVA followed by Tukey's test. $p<$ 0.05 was considered as statistically significant.

\section{Results}

\section{Lnc MT1JP is down-regulated in breast cancer cell lines}

To investigate the role of MT1JP in breast cancer, we measured the MT1JP expression in two human breast cancer cell lines (MDA-MB-231 and MCF-7) and compared the expression in these two cancer cells with a normal breast epithelial cell line, MCF-10A. Results showed that MDAMB-231 and MCF-7 cell lines had lower MT1JP expression than MCF-10A cells (Fig. 1A). These data indicate that
MT1JP expression is down-regulated in breast cancer cell lines compared with normal breast cells. Because MT1JP the low expression in MDA-MB-231 and MCF-7 cells, we generated a MT1JP-overexpressing plasmid in these two cell lines. The MT1JP expression in MDA-MB-231 cells (Fig. 1B) and MCF-7 cells (Fig. 1C) was upregulated in pcDNALnc MT1JP group, compared to cells with an empty vector (pcDNA group) and control group.

Lnc MT1JP overexpression inhibits proliferation, invasion and migration in both MDA-MB-231 and MCF-7 cells

In order to determine the effects of MT1JP to breast cancer cells on proliferation, MDA-MB-231 and MCF-7 cells were transfected with MT1JP for 24, 48, 72 and $96 \mathrm{~h}$. CCK-8 results showed that overexpression of MT1JP significantly decreased the proliferation abilities of MDA-MB-231 and MCF-7 cells after $72 \mathrm{~h}$ (Fig. 2A), and we chose $72 \mathrm{~h}$ transfection for our further study. Cell growth-associated proteins, including CDK2, cyclinE1 and P21 were analyzed in the present study. The results indicated that overexpression of MT1JP notably enhanced p21 protein expression, and weaken CDK2 and cyclinE1 protein expression in MDA-MB-231(Fig. 2B) and MCF-7 (Fig. 2C). Thus, overexpression of MT1JP significantly inhibited breast cancer cell proliferation.

Transwell assay and Scratch test were used to detect cell migration and invasion in MDA-MB-231 and MCF-7, and the results showed no significant difference between control group and pcDNA group. Overexpression of MT1JP dramatically inhibited the cell migration and invasion compared with the negative controls in the breast cancer MCF-7 and MDA-MB-231 cell lines (Fig. 3). Thus, our results suggested that MT1JP inhibited migration and invasion in breast cancer.

\section{Lnc MT1JP prevents CD151, CD147, MMP2 and MMP9 expression in breast cancer cells}

In order to explore the mechanism between Lnc MT1JP and breast cancer, cell proliferation, invasion and metasta-
A

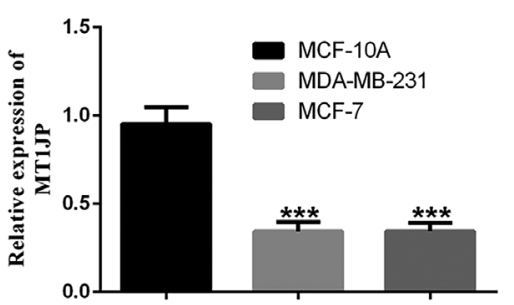

B

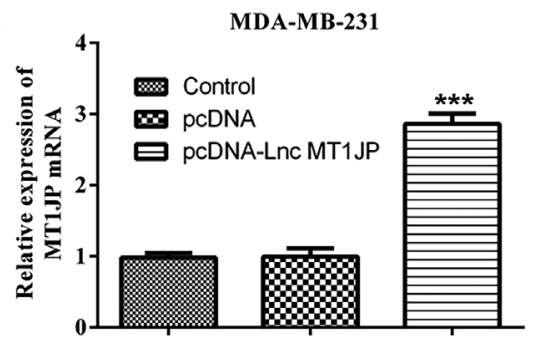

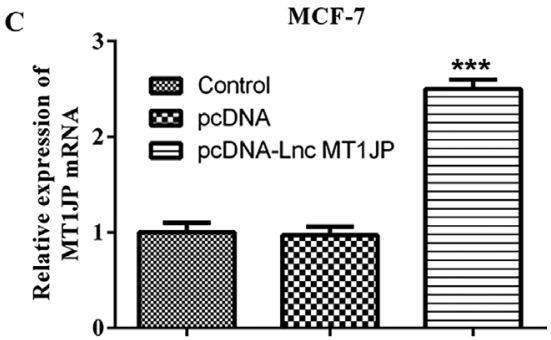

Figure 1. The expression of MT1JP in breast cancer cells. A. Relative expression levels of MT1JP in MDA-MB-231, MCF-7, and MCF-10A cells. ${ }^{* * \star} p<0.001$ vs. MCF-10A. Transfection efficacy of pcDNA-LncMT1JP in MDA-MB-231 (B) and MCF-7 (C) cells. ${ }^{* * *} p<0.001$ vs. control. Data were shown as mean $\pm \mathrm{SD}$. 
A

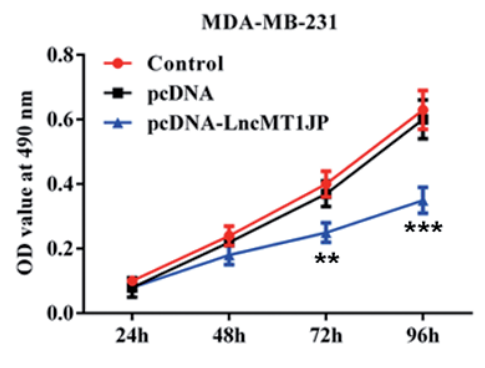

MCF-7

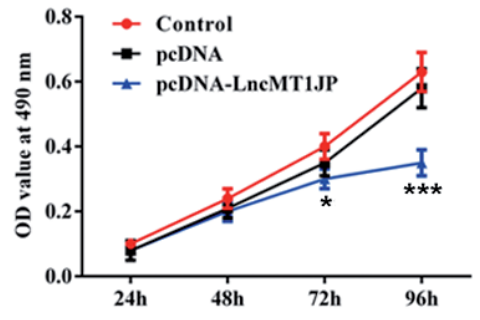

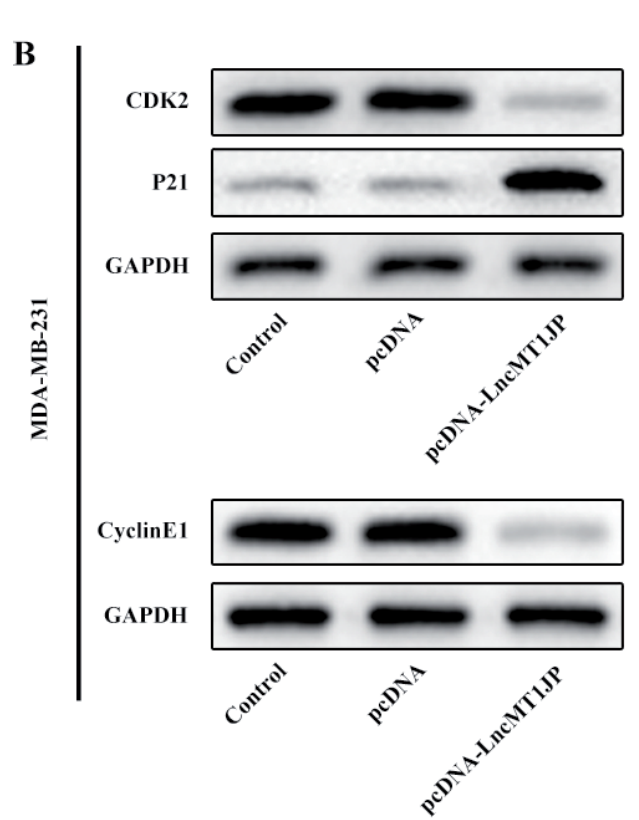
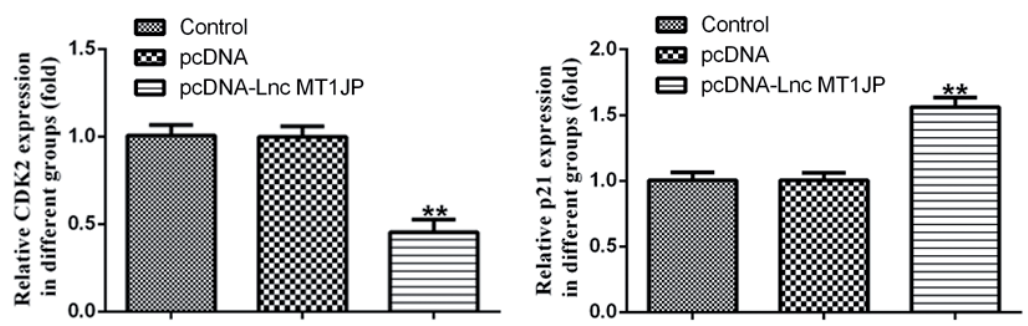

C
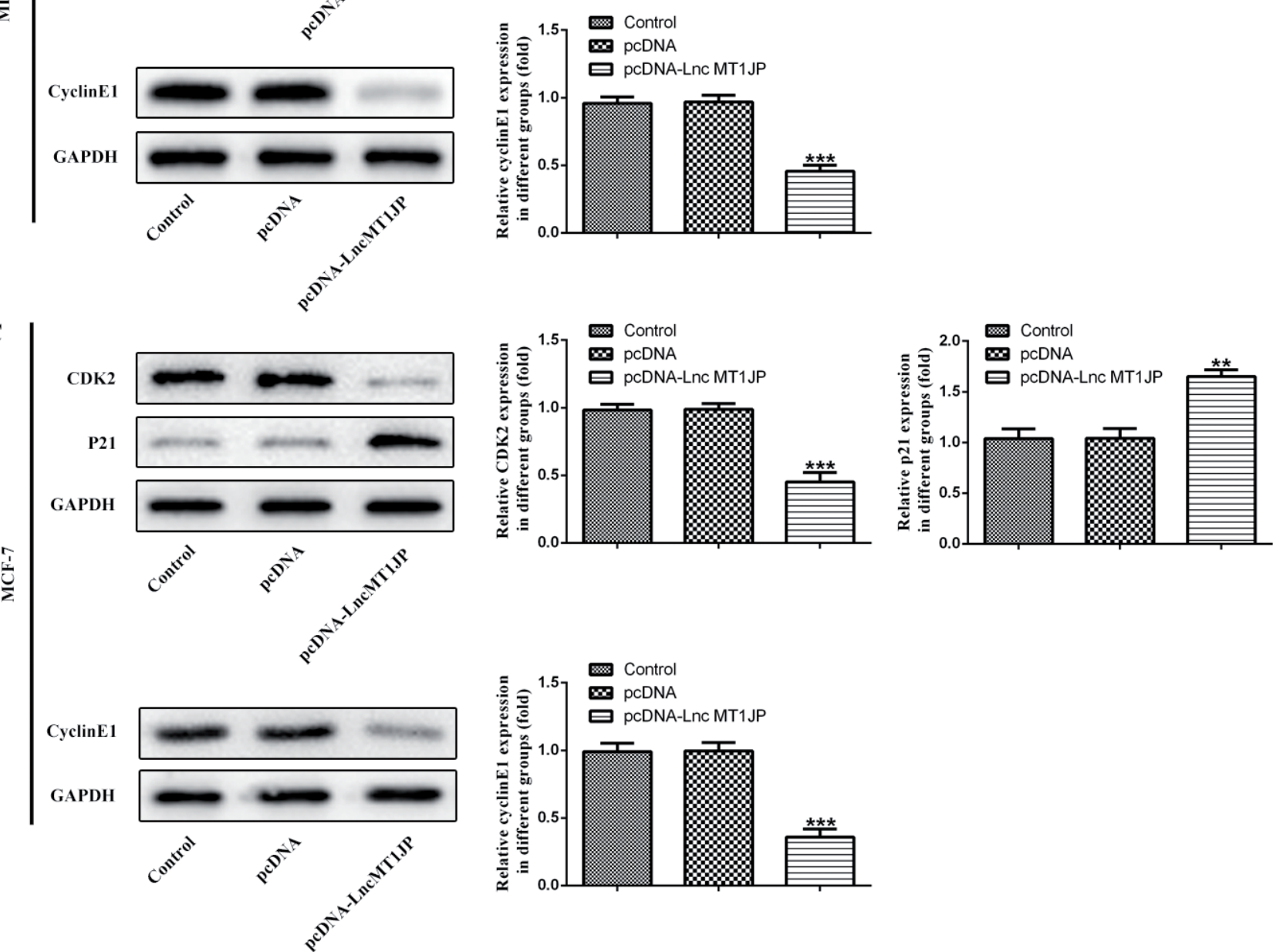

Figure 2. The effects of MT1JP on cell proliferation. A. Cell proliferation was performed at 24, 48, 72, $96 \mathrm{~h}$ using CCK8 assay. B., C. The cell cycle proteins were detected by Western blotting, and the band analysis were detected by Image J. ${ }^{\star} p<0.05,{ }^{* *} p<0.01,{ }^{* * *} p<0.001$ vs. control. Data were shown as mean $\pm \mathrm{SD}$. 
sis related proteins including CD151, CD147, MMP2 and MMP9 were analyzed in the current study. In Figure 4, our results indicated that MT1JP significantly inhibited
CD151, CD147, MMP2 and MMP9 protein expression in both MDA-MB-231 and MCF-7 cell lines compared with control and pcDNA group.
ڤ্ড

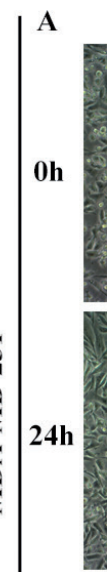

B
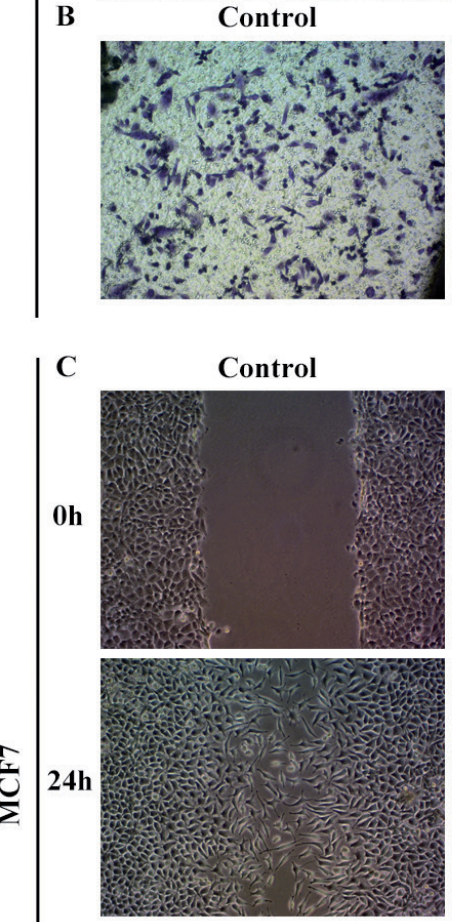

D

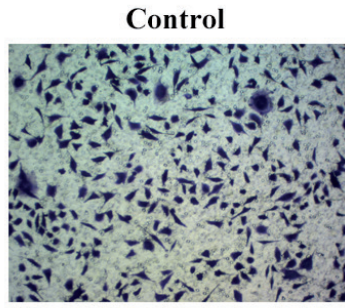

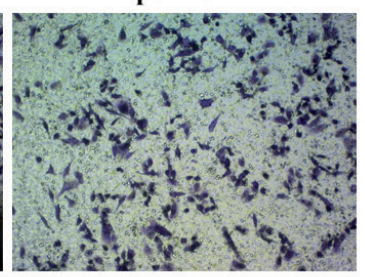

peDNA
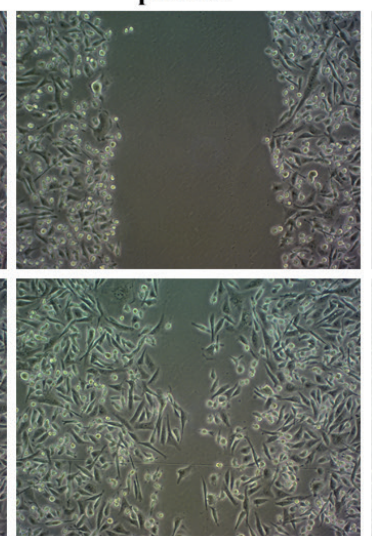

pcDNA

peDNA

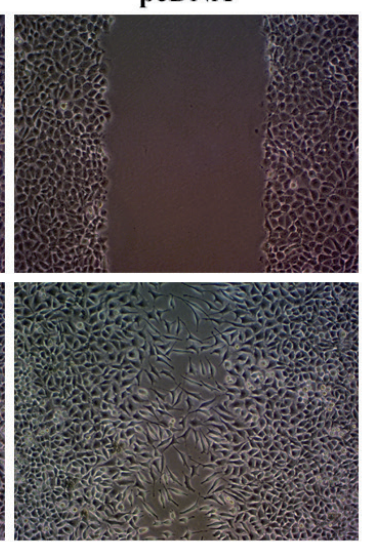

pcDNA

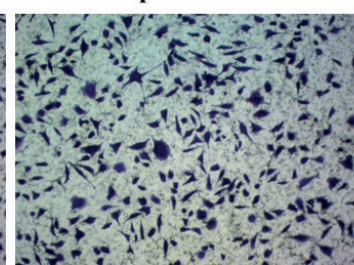

pcDNA-LncMT1JP

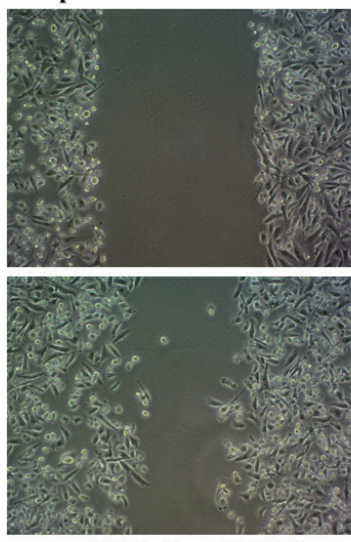

pcDNA-LncMT1JP

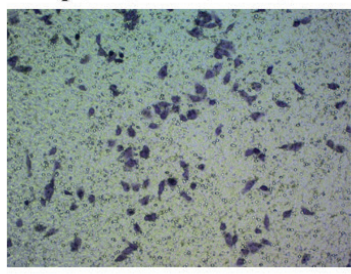

pcDNA-LncMT1JP

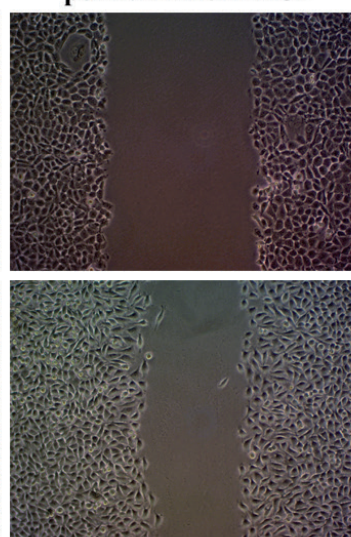

pcDNA-LncMT1JP

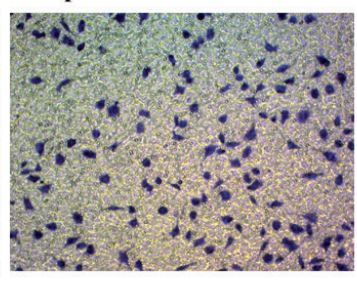

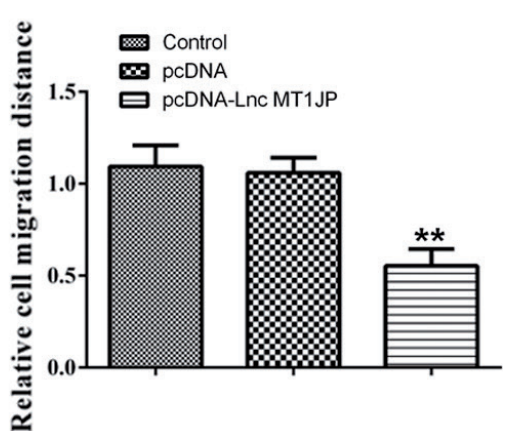

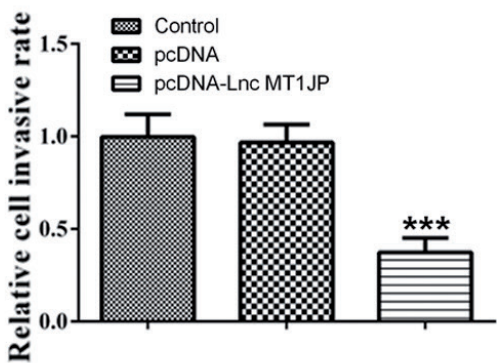

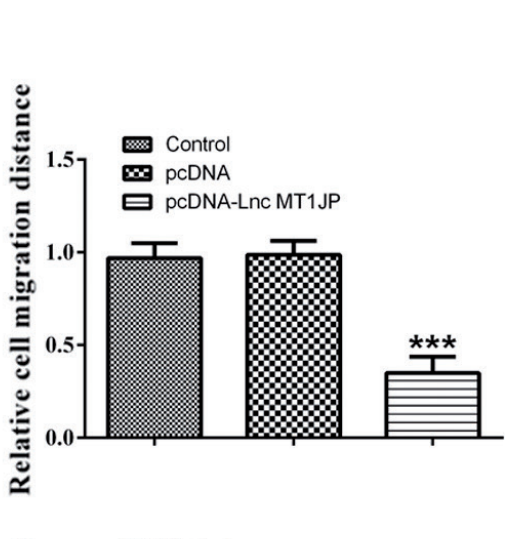

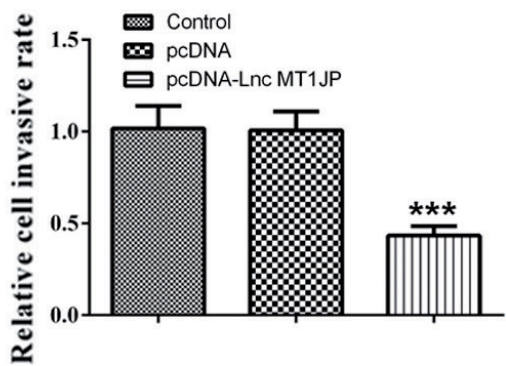

Figure 3. The effects of MT1JP on cell migration and invasion. A., C. Cell migration was assessed by wound healing assay in MDA-MB-231 and MCF-7 cells. B., D. Cell invasion was evaluated by Transwell assay in MDA-MB-231 and MCF-7 cells. ${ }^{* *} p<0.01,{ }^{* *} p<0.001 v s$. control. Data were shown as mean \pm SD. 
miR-92a-3p might be a potential mediator of Lnc MT1JP

miRNAs are crucial regulators in breast cancer. To further verify the underlying mechanism of the effect of MT1JP overexpression in breast cancer, we utilized multiple target prediction programs to identify potential MT1JP that target miRNAs. miR-92a-3p was highlighted for further investiga- tion. Figure 5 illustrated the predicted interaction between MT1JP and the miR-92a-3p.

To investigate the expression of miR-92a-3p in breast cancer cells, we measured the expression levels of miR-92a$3 \mathrm{p}$ by RT-PCR in Figure 5A, which showed an increase in MDA-MB-231 and MCF-7 cells compared with MCF-10A cells. In addition, compared with the control and the pcDNA
A

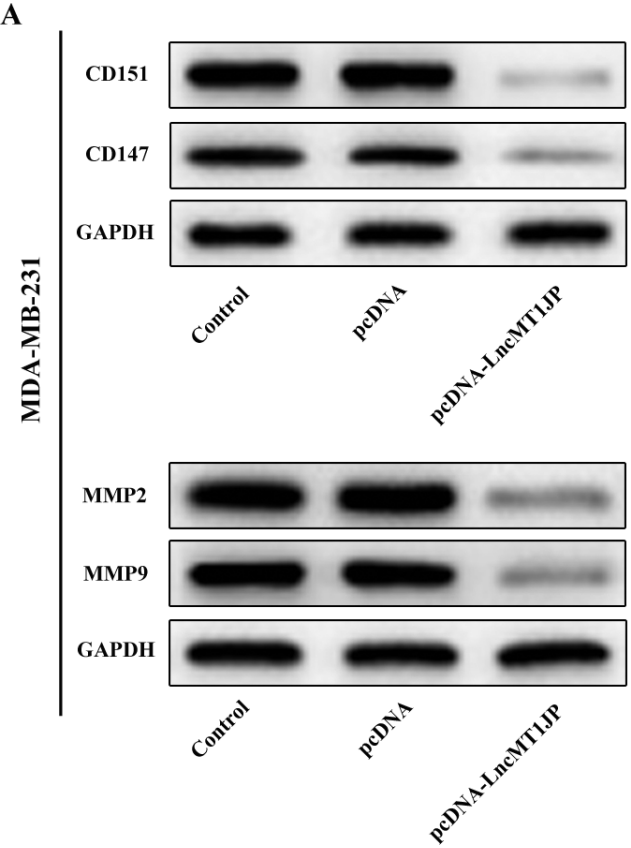

B

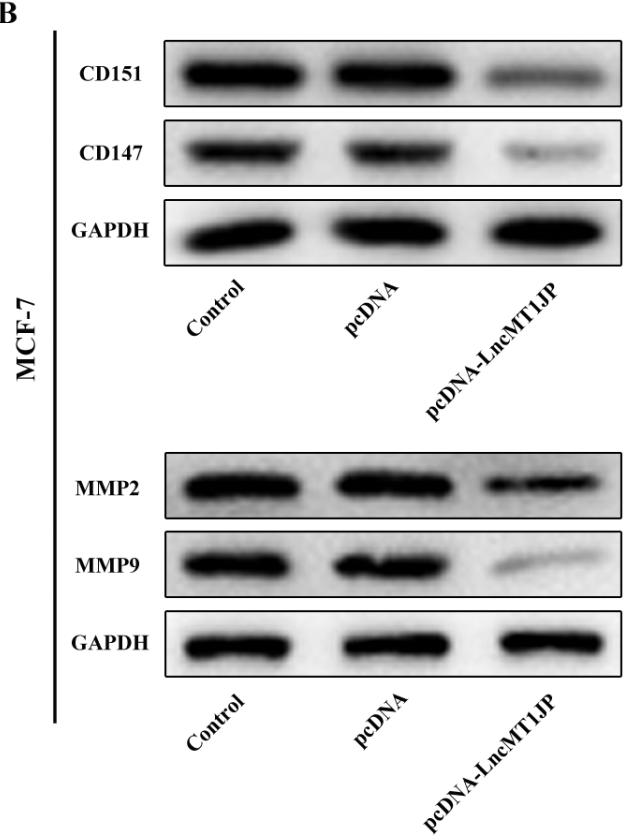

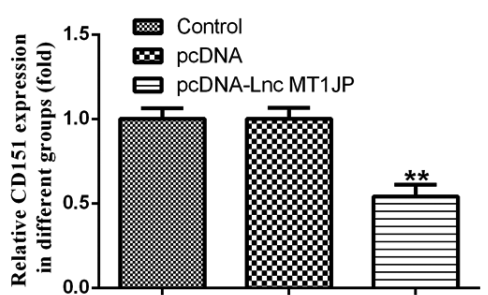
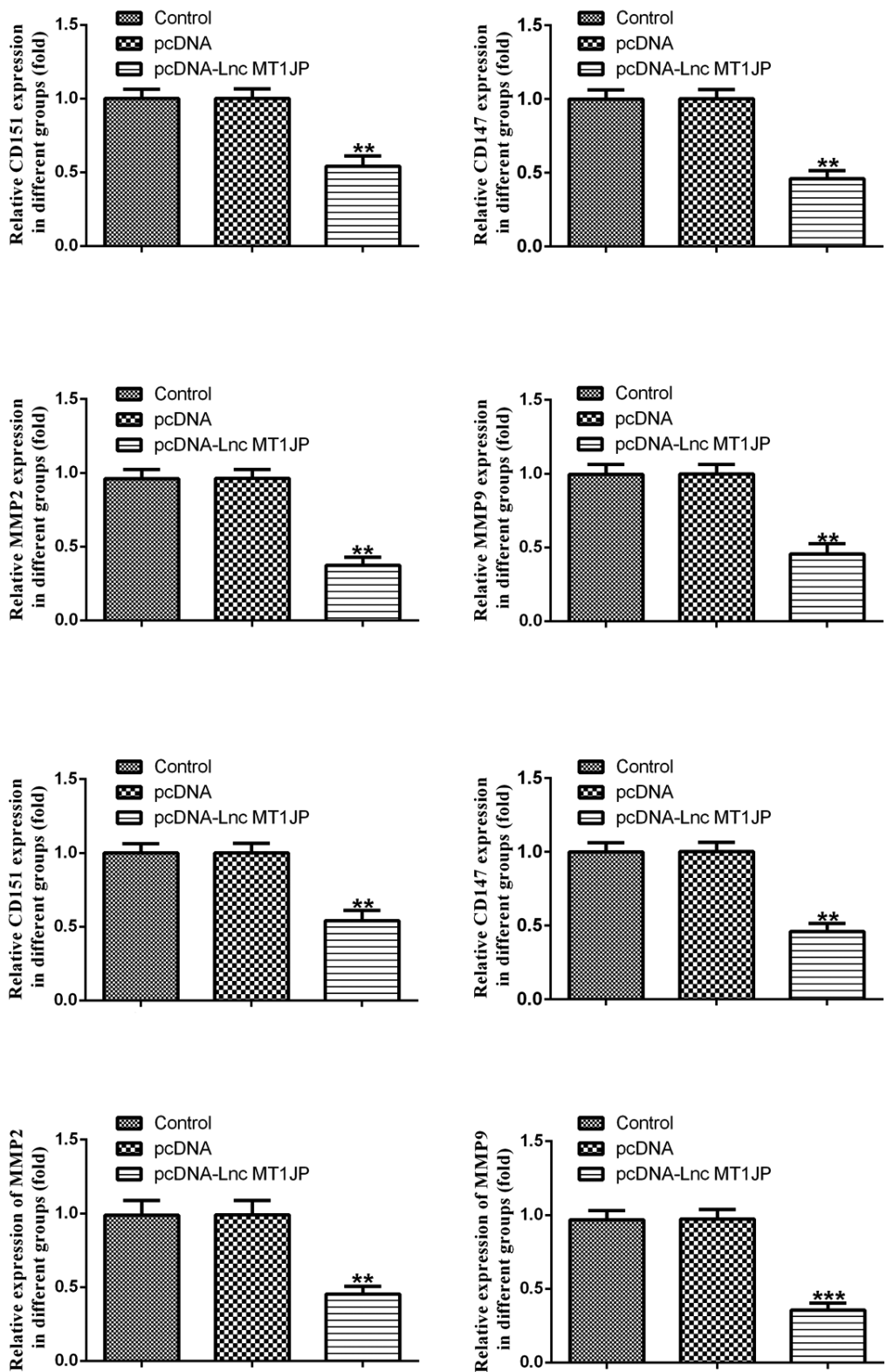

Figure 4. The effects of MT1JP on the expression levels of CD151, CD147, MMP2 and MMP9. A and B. The expression levels of CD151, CD147, MMP2 and MMP9 were detected by Western blotting. ${ }^{* *} p<0.01,{ }^{* *} p<0.001 v s$. control. Data were shown as mean $\pm \mathrm{SD}$. 
groups, the expression of miR-92a-3p significantly decreased in pcDNA-LncMT1JP group in MDA-MB-231 and MCF-7 cells, respectively (Fig. 5B and C), suggesting that MT1JP expression levels are negatively correlated with miR-92a-3p expression in breast cancer cells. These data strongly suggest that MT1JP negatively regulates miR-92-3p expression in breast cancer cells.

\section{Discussion}

In the present study, it was demonstrated that MT1JP was significantly down-regulated in breast cancer cells compared with normal breast cells. Additionally, we found that overexpression of MT1JP in breast cancer cells significantly inhibited cell proliferation, migration and invasion, and regulate the expression of CDK2, cyclin E1 and p21. To further investigated the mechanism, the data indicated that MT1JP significantly inhibited CD151, CD147, MMP2 and MMP9 protein expression in breast cancer cells. MT1JP could negatively regulate $\mathrm{miR}-92-3 \mathrm{p}$ in breast cancer cells. Therefore, our data indicate that MT1JP functions as an antitumor LncRNA by downregulating miR-92-3p, CD151 and CD147, and may serve as a novel diagnostic and therapeutic marker of breast cancer. Due to the effects of MT1JP in both MDA-MB-231 (Triplicate-negative breast cancer) and MCF-7 (ER-positive breast cancer) cells were not differences, therefore, LncRNA MT1JP is not an ER-specific target for breast cancer treatment.

It has been reported that breast cancer is the most common cause of death among women, and more than one million women diagnosed with breast cancer annually (Igene 2008). Therefore, it is urgent to find novel markers that could serve as an early diagnostic and therapeutic target for breast cancer. A large number of evidences suggest that LncRNAs are important to the regulation of cell differentiation, proliferation, and apoptosis (Tay et al. 2014). It is also known that aberrant expression of LncRNAs have crucial roles in the initiation and development of various human cancers, including breast cancer (Youness and Gad 2019). In this study, we found that MT1JP expression was down-regulated in breast cancer cell lines. We also found that MT1JP overexpression significantly inhibited proliferation, migration and invasion of breast cancer cells. These data indicated that MT1JP could serve as a novel biomarker for breast cancer diagnosis, and acted as a tumor suppressor in breast cancer, which is consistent with previous studies that reported tumor-suppressing functions for MT1JP in gastric cancer.

As a tetraspanin family member, CD151 modulates the ligand binding activity of integrin $\alpha 3 \beta 1$. CD 151 promote metastasis of cancer cells. Also, CD151 is reported to play a role in Met-dependent signaling and the TGF- $\beta$ signaling (Sadej et al. 2010). In breast cancer, CD151 overexpression was found to be significantly associated with larger tumor size, higher nodal stage and advanced stage (Kwon et al. 2012). Previous studies confirmed that miR-124 inhibited breast cancer cell growth by targeting CD151. MiR-124/CD151 axis contributed to the proliferation and migration of breast cancer cells (Han et al. 2013). The current data showed that overexpression of MT1JP dramatically decreased the expression of CD151, suggesting that CD151 might be involved in the process of MT1JP inhibiting breast cancer proliferation, migration and invasion. And another study suggested that LncRNA SNHG3 induced EMT and sorafenib resistance by modulating the miR-128/ CD151 pathway in hepatocellular carcinoma (Zhang et al. 2019). CD147 is a matrix metalloproteinase (MMP) inducer that is performed to process cancer progression (Sun and Hemler 2001; Rucci et al. 2010). MMP family proteins such as MMP2, -9 -11 and -14 have been elucidated to degrade extracellular matrix (ECM) to promote tumor metastasis and invasion (Cancemi et al. 2019). The results manifested that MT1JP inhibits expression of CD147 as well as the MMP2 and MMP9, indicating that MT1JP might regulate breast cancer malignant by ECM remodeling. However, further studies still require exploring the relationship and mechanism among LncRNA MT1JP, CD151 and CD147.
A

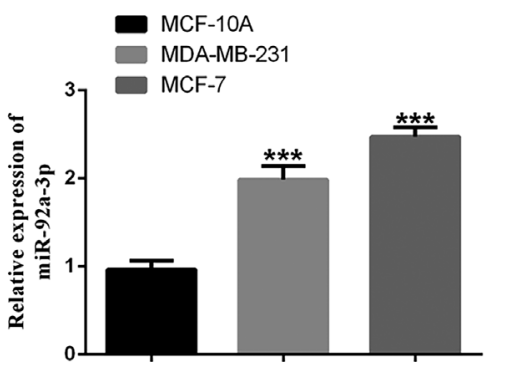

B

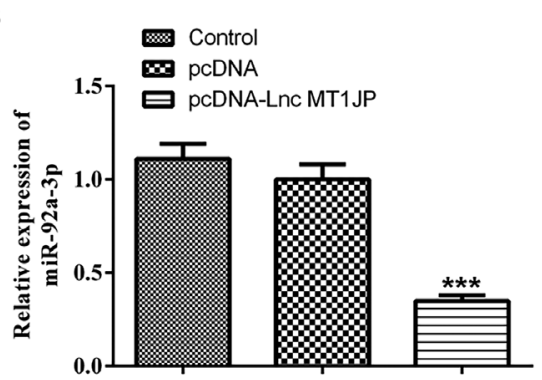

C

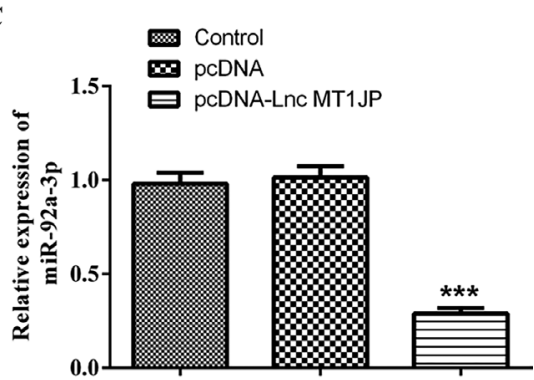

Figure 5. The effects of MT1JP on the expression of miR-92a-3p. A. Relative expression levels of miR-92a-3p in MDA-MB-231 and MCF-7 cells ( ${ }^{* * *} p<0.001 v s$. MCF-10A). Relative expression levels of miR-92a-3p in MDA-MB-231 (B) and MCF-7 (C) cells overexpressed MT1JP. ${ }^{* * *} p<0.001 v s$. control. Data were shown as mean \pm SD. 
miRNAs are a group of small, highly conserved non-coding RNA molecule, which acted as regulators in the tumorigenesis, carcinogenesis, and tumor angiogenesis (Croce 2009). And miR-92a-3p belongs to the miR-17-92 family that was reported to highly expressed in colorectal carcinoma, hepatocellular carcinoma, and esophageal squamous cell carcinoma (Shigoka et al. 2010; Chen et al. 2011; Tsuchida et al. 2011). In addition, miR-92a-3p may be used as a potential biomarker for early detection of cancer and monitoring the efficacy of endocrine therapy by bioinformatics-based interaction analysis (Cun and Yang 2018). Recently, novel regulatory mechanisms concern about LncRNAs and miRNAs have been revealed in cancer development. LncRNA can regulate miRNAs by acting as molecular sponges or ceRNA. Previous study found that LncRNA MALAT1 promoted growth and metastasis of bladder transitional cell carcinoma by targeting miR-124 (Jiao et al. 2018). SNHG14 and miR-92a-3p may be promising molecular targets for glioma therapy (Wang et al. 2018). MT1JP also regulated the progression of gastric cancer by competitively binding to miR-92a-3p and regulating FBXW7 expression (Zhang et al. 2018). Previous investigation has been indicated that miR-92 expression were increased in breast cancers compare with the corresponding normal control (Sempere et al. 2007), and downregulation of miR-92 could promote ER $\beta 1$ which is a tumor suppressor in breast cancer (Al-Nakhle et al. 2010). In present study, we confirmed MT1JP could negatively regulate miR-92-3p in breast cancer cells, which was consistent with the previous study in gastric cancer. Therefore, MT1JP effects on breast cancer cell might mediate by repressing miR-92-3p, and the mechanism of MT1JP and miR-92a-3p in breast cancer remains to be investigated.

In summary, we found that MT1JP was significantly down-regulated in breast cells. Overexpression of MT1JP in breast cancer cells significantly inhibited cell proliferation, migration and invasion. The mechanism for these effects is MT1JP binding and repressing miR-92-3p, thereby may inhibiting the expression of CD151. This study provides a novel insight that could aid the development of potential prognostic indicators for breast cancer patients and improve the breast cancer treatment.

\section{References}

Al-Nakhle H, Burns PA, Cummings M, Hanby AM, Hughes TA, Satheesha S, Shaaban AM, Smith L, Speirs V (2010): Estrogen receptor $\{$ beta 1 expression is regulated by miR-92 in breast cancer. Cancer Res. 70, 4778-4784 https://doi.org/10.1158/0008-5472.CAN-09-4104

Burki TK (2018): Responses to carboplatin in BRCA1/2-mutated breast cancer. Lancet Oncol. 19, e337 https://doi.org/10.1016/S1470-2045(18)30407-8

Cancemi P, Buttacavoli M, Roz E, Feo S (2019): Expression of alphaenolase (ENO1), myc promoter-binding protein-1 (MBP-1) and matrix metalloproteinases (MMP-2 and MMP-9) reflect the nature and aggressiveness of breast tumors. Int. J. Mol. Sci. 20, e3952 https://doi.org/10.3390/ijms20163952

Chen ZL, Zhao XH, Wang JW, Li BZ, Wang Z, Sun J, Tan FW, Ding DP, Xu XH, Zhou F, et al. (2011): microRNA-92a promotes lymph node metastasis of human esophageal squamous cell carcinoma via E-cadherin. J. Biol. Chem. 286, 10725-10734 https://doi.org/10.1074/jbc.M110.165654

Croce CM (2009): Causes and consequences of microRNA dysregulation in cancer. Nat. Rev. Genet. 10, 704-714 https://doi.org/10.1038/nrg2634

Cun J, Yang Q (2018): Bioinformatics-based interaction analysis of miR-92a-3p and key genes in tamoxifen-resistant breast cancer cells. Biomed. Pharmacother. 107, 117-128 https://doi.org/10.1016/j.biopha.2018.07.158

Han ZB, Yang Z, Chi Y, Zhang L, Wang Y, Ji Y, Wang J, Zhao H, Han ZC (2013): microRNA-124 suppresses breast cancer cell growth and motility by targeting CD151. Cell. Physiol. Biochem. 31, 823-832 https://doi.org/10.1159/000350100

Igene H (2008): Global health inequalities and breast cancer: an impending public health problem for developing countries. Breast J. 14, 428-434 https://doi.org/10.1111/j.1524-4741.2008.00618.x

Jastrzebski K, Thijssen B, Kluin RJC, de Lint K, Majewski IJ, Beijersbergen RL, Wessels LFA (2018): Integrative modeling identifies key determinants of inhibitor sensitivity in breast cancer cell lines. Cancer Res. 78, 4396-4410 https://doi.org/10.1158/0008-5472.CAN-17-2698

Jiao D, Li Z, Zhu M, Wang Y, Wu G, Han X (2018): LncRNA MALAT1 promotes tumor growth and metastasis by targeting miR-124/foxq1 in bladder transitional cell carcinoma (BTCC). Am. J. Cancer Res. 8, 748-760

Kwon MJ, Park S, Choi JY, Oh E, Kim YJ, Park YH, Cho EY, Kwon MJ, Nam SJ, Im YH, et al. (2012): Clinical significance of CD151 overexpression in subtypes of invasive breast cancer. Br. J. Cancer 106, 923-930 https://doi.org/10.1038/bjc.2012.11

Li T, Meng XL, Yang WQ (2017): Long noncoding RNA PVT1 acts as a "sponge" to inhibit microRNA-152 in gastric cancer cells. Dig. Dis. Sci. 62, 3021-3028 https://doi.org/10.1007/s10620-017-4508-Z

Li Y, Xu J, Chen L, Zhong WD, Zhang Z, Mi L, Zhang Y, Liao CG, Bian HJ, Jiang JL, et al. (2009): HAb18G (CD147), a cancerassociated biomarker and its role in cancer detection. Histopathology 54, 677-687 https://doi.org/10.1111/j.1365-2559.2009.03280.x

Liu J, Song Z, Feng C, Lu Y, Zhou Y, Lin Y, Dong C (2017): The long non-coding RNA SUMO1P3 facilitates breast cancer progression by negatively regulating miR-320a. Am. J. Transl. Res. 9, 5594-5602

Liu L, Yue H, Liu Q, Yuan J, Li J, Wei G, Chen X, Lu Y, Guo M, Luo J, Chen R (2016): LncRNA MT1JP functions as a tumor suppressor by interacting with TIAR to modulate the $\mathrm{p} 53$ pathway. Oncotarget 7, 15787-15800 https://doi.org/10.18632/oncotarget.7487

Liu T, Wang S, Wang L, Wang J, Li Y (2015): Targeting CD151 by lentivirus-mediated RNA interference inhibits luminal and 
basal-like breast cancer cell growth and invasion. Mol. Cell. Biochem. 407, 111-121 https://doi.org/10.1007/s11010-015-2459-2

Liu X, Bi L, Wang Q, Wen M, Li C, Ren Y, Jiao Q, Mao JH, Wang C, Wei G, Wang Y (2018): miR-1204 targets VDR to promotes epithelial-mesenchymal transition and metastasis in breast cancer. Oncogene 37, 3426-3439 https://doi.org/10.1038/s41388-018-0215-2

Livak KJ, Schmittgen TD (2001): Analysis of relative gene expression data using real-time quantitative PCR and the 2(-Delta Delta C(T)) method. Methods 25, 402-408 https://doi.org/10.1006/meth.2001.1262

Lu L, Dong J, Wang L, Xia Q, Zhang D, Kim H, Yin T, Fan S, Shen Q (2018): Activation of STAT3 and Bcl-2 and reduction of reactive oxygen species (ROS) promote radioresistance in breast cancer and overcome of radioresistance with niclosamide. Oncogene 37, 5292-5304 https://doi.org/10.1038/s41388-018-0340-y

Lv Z, Zhang Y, Yu X, Lin Y, Ge Y (2018): The function of long noncoding RNA MT1JP in the development and progression of gastric cancer. Pathol. Res. Pract. 214, 1218-1223 https://doi.org/10.1016/j.prp.2018.07.001

Nagini S (2017): Breast cancer: current molecular therapeutic targets and new players. Anticancer Agents Med. Chem. 17, 152-163 https://doi.org/10.2174/1871520616666160502122724

Pinheiro C, Garcia EA, Morais-Santos F, Moreira MA, Almeida FM, Jube LF, Queiroz GS, Paula EC, Andreoli MA, Villa LL, et al. (2015): Reprogramming energy metabolism and inducing angiogenesis: co-expression of monocarboxylate transporters with VEGF family members in cervical adenocarcinomas. BMC Cancer 15, 835 https://doi.org/10.1186/s12885-015-1842-4

Rucci N, Millimaggi D, Mari M, Del Fattore A, Bologna M, Teti A, Angelucci A, Dolo V (2010): Receptor activator of NF-kappaB ligand enhances breast cancer-induced osteolytic lesions through upregulation of extracellular matrix metalloproteinase inducer/CD147. Cancer Res. 70, 6150-6160 https://doi.org/10.1158/0008-5472.CAN-09-2758

Sadej R, Romanska H, Kavanagh D, Baldwin G, Takahashi T, Kalia N, Berditchevski F (2010): Tetraspanin CD151 regulates transforming growth factor beta signaling: implication in tumor metastasis. Cancer Res. 70, 6059-6070 https://doi.org/10.1158/0008-5472.CAN-09-3497

Sempere LF, Christensen M, Silahtaroglu A, Bak M, Heath CV, Schwartz G, Wells W, Kauppinen S, Cole CN (2007): Altered microRNA expression confined to specific epithelial cell subpopulations in breast cancer. Cancer Res. 67, 11612-11620 https://doi.org/10.1158/0008-5472.CAN-07-5019

Shigoka M, Tsuchida A, Matsudo T, Nagakawa Y, Saito H, Suzuki Y, Aoki T, Murakami Y, Toyoda H, Kumada, et al. (2010): Deregulation of miR-92a expression is implicated in hepatocellular carcinoma development. Pathol. Int. 60, 351-357 https://doi.org/10.1111/j.1440-1827.2010.02526.x

Siegel RL, Miller KD, Jemal A (2017): Cancer Statistics, 2017. CA Cancer J. Clin. 67, 7-30 https://doi.org/10.3322/caac.21387

Sun J, Hemler ME (2001): Regulation of MMP-1 and MMP-2 production through CD147/extracellular matrix metalloproteinase inducer interactions. Cancer Res. 61, 2276-2281
Tay Y, Rinn J, Pandolfi PP (2014): The multilayered complexity of ceRNA crosstalk and competition. Nature 505, 344-352 https://doi.org/10.1038/nature12986

Thirumamagal R, Fowziva SA, Uduman Mohideen AM, Hameedha Beevi A, Ayeshamariam A, Mohamed Saleem A (2018): Evaluation of the cytotoxicity effect on HAp doped with Ce2O3 and its assessment with breast cancer. Cell Line of MCF-7. J. Bionanosci. 12, 350-356 https://doi.org/10.1166/jbns.2018.1530

Thomson DW, Dinger ME (2016): Endogenous microRNA sponges: evidence and controversy. Nat. Rev. Genet. 17, 272-283 https://doi.org/10.1038/nrg.2016.20

Tsuchida A, Ohno S, Wu W, Borjigin N, Fujita K, Aoki T, Ueda S, Takanashi M, Kuroda M (2011): miR-92 is a key oncogenic component of the miR-17-92 cluster in colon cancer. Cancer Sci. 102, 2264-2271 https://doi.org/10.1111/j.1349-7006.2011.02081.x

Ulker D, Ersoy YE, Gucin Z, Muslumanoglu M, Buyru N (2018): Downregulation of SCARA5 may contribute to breast cancer via promoter hypermethylation. Gene 673, 102-106 https://doi.org/10.1016/j.gene.2018.06.036

Wang Q, Teng Y, Wang R, Deng D, You Y, Peng Y, Shao N, Zhi F (2018): The long non-coding RNA SNHG14 inhibits cell proliferation and invasion and promotes apoptosis by sponging miR-92a-3p in glioma. Oncotarget 9, 12112-12124 https://doi.org/10.18632/oncotarget.23960

Xu Y, Zhang G, Zou C, Zhang H, Gong Z, Wang W, Ma G, Jiang P, Zhang W (2018): LncRNA MT1JP suppresses gastric cancer cell proliferation and migration through MT1JP/MiR-214-3p/ RUNX3 Axis. Cell. Physiol. Biochem. 46, 2445-2459 https://doi.org/10.1159/000489651

Youness RA, Gad MZ (2019): Long non-coding RNAs: Functional regulatory players in breast cancer. Noncoding RNA Res. 4, 36-44 https://doi.org/10.1016/j.ncrna.2019.01.003

Zhang G, Li S, Lu J, Ge Y, Wang Q, Ma G, Zhao Q, Wu D, Gong W, Du M, et al. (2018): LncRNA MT1JP functions as a ceRNA in regulating FBXW7 through competitively binding to miR-92a$3 \mathrm{p}$ in gastric cancer. Mol. Cancer 17, 87 https://doi.org/10.1186/s12943-018-0829-6

Zhang PF, Wang F, Wu J, Wu Y, Huang W, Liu D, Huang XY, Zhang XM, Ke AW (2019): LncRNA SNHG3 induces EMT and sorafenib resistance by modulating the miR-128/CD151 pathway in hepatocellular carcinoma. J. Cell. Physiol. 234, 2788-2794 https://doi.org/10.1002/jcp.27095

Zhang XA, Kazarov AR, Yang X, Bontrager AL, Stipp CS, Hemler ME (2002): Function of the tetraspanin CD151-alpha6beta1 integrin complex during cellular morphogenesis. Mol. Biol. Cell. 13, 1-11

https://doi.org/10.1091/mbc.01-10-0481

Zhu C, Ma J, Li Y, Zhang Y, Da M (2018): Low expression of long noncoding RNA MT1JP is associated with poor overall survival in gastric cancer patients: Protocol for meta-analysis. Medicine (Baltimore) 97, e10394 https://doi.org/10.1097/MD.0000000000010394

Received: July 30, 2019

Final version accepted: September 14, 2019 